\title{
Uma Análise de Performance de Cinco Fundos de Investimento Mobiliário Harmonizados de Ações Portuguesas ${ }^{1}$
}

\author{
Henrique Amaral Dias \\ Instituto Superior Miguel Torga - henriqueamaraldias@gmail.com
}

\begin{abstract}
Sumário
Este artigo analisa cinco Fundos de Investimento Mobiliário (F.I.M.) Harmonizados de Ações Portuguesas através de duas perspetivas, com os seguintes objetivos e metodologias: i) calcular a pior perda a que um investidor estaria sujeito com um nível de confiança de 99\%, tendo para tal utilizado o VaR Histórico ii) verificar quais os F.I.M. que obtiveram uma performance superior/inferior à do mercado em termos da taxa média de retorno anual, quantificando essas diferenças. Por outro lado, estabelecer uma relação entre rendibilidade e risco, ao longo de um período de cinco anos. Para tal aplicámos o CAPM (Capital Asset Pricing Model) e os indicadores relevantes (Alpha

de Jensen e os Rácios de Treynor e de Sharpe).

Os resultados permitiram-nos uma hierarquização dos fundos aplicando os critérios mencionados, a partir das suas performances históricas: i) o do NB Portugal foi o que sofreu as menos severas piores perdas ii) o do Santander foi o que registou a maior diferença entre a sua taxa média geométrica de retorno anual e a do respetivo benchmark iii) o do BPI apresentou a maior diferença entre rendibilidades por unidade de risco.

Os investidores devem considerar resultados análogos na tomada de decisão e recomenda-se que as autoridades reguladoras publiquem métricas similares regularmente.
\end{abstract}

Palavras-chave: VaR, CAPM, Rácio de Sharpe, Fundos de Investimento

\section{A Performance Analysis of Five Harmonized Portuguese Equity Investment Funds}

\begin{abstract}
This paper examines five harmonized Portuguese equity investment funds (PEIF) through two distinct perspetives, with the following objetives and methodologies i) to compute the worst loss for an investor with a confidence level of $99 \%$. In order to do so we have used

the Historical VaR. ii) to assess the investment funds that have outperformed/underperformed the market in terms of their yearly average rate of return and by how much, and to establish a relation between profitability and risk regarding the investment funds consid-

1 Este trabalho deve-se, prima facie, ao Prof. Doutor Carlos Francisco Alves pelos conhecimentos ministrados na disciplina de Modelos de Avaliação de Ativos da Pós-Graduação em Análise Financeira da Porto Business School e orientação proporcionada.
\end{abstract}


ered, during a period of five years. With these aims we used the CAPM (Capital Asset Pricing Model) and its relevant indicators (Jensen's Alpha, Treynor and Sharpe Ratios).

The results enabled us to hierarchize the funds applying the above criteria, given their past performances: i) the NB Portugal PEIF suffered the less severe worst losses ii) the San- tander PEIF had the largest difference between its yearly average geometric rate of return and the respective benchmark rate iii) the BPI PEIF generated the greatest excess of return per unit of risk. Investors should take into account analogous results and the regulatory authorities should publish similar metrics on a systematic basis.

Keywords: VaR, CAPM, Sharpe Ratio, Investment Funds

\section{INTRODUÇÃO}

\section{Considerações sobre o VaR (Value at Risk)}

Esta metodologia tenta responder a uma questão essencial, tanto para investidores não profissionais, como para os mais sofisticados: "Qual a maior perda a que posso estar sujeito com este investimento?" As três mais generalizadas medidas do VaR são: o VaR Paramétrico, o VaR de Monte Carlo e a por nós escolhida, o VaR Histórico. Esta último não está sujeito a uma distribuição normal, o que é uma vantagem tendo em vista o que as experiências passadas demonstraram. Sucintamente, ordenamos os retornos históricos do menor para o maior e calculamos, para um pré-determinado nível de confiança, qual foi o menor retorno historicamente registado. Por outro lado, sem uma distribuição atribuída, está a assumir-se que o passado replicará o futuro. Sabemos que se trata de uma hipótese forçada. Como Cícero afirmou (45 a.C./1923, Liber primus, LVI, 127):

"Aquele que conhece as causas dos eventos futuros sabe necessariamente todo e cada acontecimento futuro. Mas, uma vez que tal conhecimento é possível apenas para um deus, ao homem resta pressagiar o destino recorrendo a determinados sinais que indicam o que se lhes seguirá."

Embora este método esteja sob escrutínio severo, sobretudo desde a crise financeira que emergiu em $2007^{2}$, o sistema financeiro continua a aplicá-lo. Diversos aca-

2 Como é apontado por Youngman (2009, p. 52): “Os modelos de Value-at-Risk padecem de limitações amplamente reconhecidas e têm estado sujeitos a severas críticas tanto por académicos, como por utilizadores. Pese embora as entidades bancárias tenham desenvolvido diversas variantes dos modelos VaR, todos eles ainda assentam em dados históricos para estimar a distribuição de probabilidade de resultados futuros. A 
démicos têm vindo a propor testes de validação e variações do VaR para aperfeiçoar as suas capacidades preditivas. Hurlin e Tokpavi (2007) desenvolveram um novo teste da validação do VaR, nomeadamente mostram que a alteração para uma dimensão multivariável melhora o poder das propriedades do teste de validação do VaR para amostras de dimensão adequada. Também Mitrodima e Oberoi (2017) aplicaram diversas melhorias:

"na performance financeira dos modelos de CAViaR existentes através da introdução de propriedades de memória longa, do uso de uma estratégia alternativa de estimação e, finalmente, pela inclusão de informação em retornos de múltiplos horizontes entre os preditores do VaR." (2017, p. 28)

Apesar das críticas, o nosso objetivo foi identificar os F.I.M. mais sujeitos às maiores perdas, com base no seu comportamento passado, o que necessariamente reflete a abordagem ao risco adotada pelos seus gestores. Ceteris paribus (mesmos gestores, mesma estratégia, mesmos ativos, etc.), o VaR é um indicador (ainda que imperfeito) da qualidade relativa futura da gestão no que concerne à minimização das maiores perdas a suportar pelos investidores. Mesmo que sujeito às palavras cautelares de Cícero, tudo o resto constante, um investidor deverá preferir o investimento com o menor VaR.

Consequente e justificadamente, medimos como os F.I.M. se comportaram aplicando o VaR, não com o intento de afirmar que este método é isento de falhas ou exato, mas como uma medida de performance que pode ser objetiva e transversalmente aplicada quanto o investidor toma a decisão e que, portanto, não deve por ele ser ignorada.

maioria dessas entidades utilizam um período relativamente curto de dados históricos (the "lookback period") para estimar a probabilidade de fatores de Mercado, e alguns recorrem a ponderadores, de modo a que, no lookback period, os dados mais recentes tenham um fator ponderador mais elevado. Estas técnicas asseguram que os VaRs estimados refletem com precisão o facto estilizado que muitas das séries temporais exibem uma volatilidade sensível ao tempo. Neste sentido, tais modelos VaR são "sensíveis ao risco", na medida em que relacionam capital a estimativas correntes de risco. Esta sensibilidade ao risco faz com que os VaRs sejam cíclicos: aumentando e diminuindo com a volatilidade do mercado."

(Taleb, 2008, p. 298): "Surpreendentemente a curva em forma de sino é usada como ferramenta de medição do risco pelos reguladores e banqueiros centrais (...). E mais adiante na p. 302: "Na matriz gaussiana a desigualdade desce à medida que os desvios se avolumam - provocados pela taxa de decréscimo."

(Blyth, 2013, p. 65): "O VaR e as técnicas associadas tomam o passado como amostra para prever o futuro e dessa informação derivamos teorias acerca do modo como o futuro devia desenrolar-se com base nas nossas expectativas da distribuição de probabilidade e não nas nossas experiências reais do mundo.” 
Uma Análise de Performance de Cinco Fundos de Investimento Mobiliário Harmonizados de Ações Portuguesas

Não obstante, um investidor prudente não deverá olvidar que o pior cenário registado não será o futuro pior cenário, caso contrário arrisca-se a ser confundido com David A. Viniar, CFO do Goldman Sachs até janeiro de 2013, que afirmou em 2007: "Estamos a observar variações de 25 desvios-padrão, em dias sucessivos." (citado por Dowd, Cotter, Humphrey, \& Woods, 2008, p. 1). Mas como é consabido "a probabilidade de ocorrência de um evento 25 sigma é comparável à probabilidade de acertar na lotaria 21 ou 22 vezes consecutivamente." (Dowd, Cotter, Humphrey, \& Woods, 2008, p. 3).

\section{Considerações sobre o CAPM (Capital Asset Pricing Model)}

Partindo do CAPM (Markowitz, 1952; Lintner, 1965; Sharpe, 1964; Merton, 1973), o Alpha de Jensen dá-nos o excesso de rendibilidade face ao benchmark selecionado, o que é uma medida de performance relevante quando estamos a comparar F.I.M. similares. Contudo, este indicador é uma medida absoluta, uma vez que não considera o risco incorrido por cada F.I.M. Assim, e mais importante, temos de relacionar o excesso de rendibilidade com indicadores de risco. Deste modo, os rácios de Sharpe e de Treynor foram utilizados para precisamente aquilatar dessa relação. E tal conduz-nos à questão de saber quais os F.I.M. que geraram maiores excessos médios de retorno por unidade de risco assumida, utilizando para tal no denominador o desvio-padrão. O rácio de Sharpe tem uma vantagem sobre o de Treynor, uma vez que adota o risco total e não apenas o risco sistemático. Um investidor experimentado deve, pois, considerar que o rácio de Sharpe é um indicador mais adequado. Posto que estamos a considerar fundos de ações, uma amostra que abranja um horizonte temporal de curto prazo não é simplesmente adequada.

Apesar das objeções fundamentadas de Fama e French (2004, p. 44) ainda assim consideram o CAPM "um tour de force teórico", embora "fundos que se concentrem em ações de beta reduzido (...) tenderão a produzir retornos positivos anormais com relação às previsões do CAPM de Sharpe-Lintner, mesmo quando os seus gestores não possuem qualquer talento especial na seleção de ações vencedoras.".

No mesmo artigo, Fama e French (2004, p. 41), apontam mais uma insuficiência ao CAPM de Sharpe-Lintner:

"Stambough (1982) testa o CAPM selecionando uma gama de portefólios de mercado que incluem, além de ações norte-americanas ordinárias, obrigações 
corporate e governamentais, ações preferenciais, imóveis e outros bens de consumo duradouro. Chega à conclusão que os testes do CAPM não são sensíveis à aplicação de uma proxy de mercado que abranja mais do que ações ordinárias."

Todavia, o CAPM é ainda assim muito útil e aplicado em circunstâncias semelhantes quando se trata de ações ordinárias ou setores específicos, tal como a indústria dos bancos comerciais norte-americanos (DeYoung, Kowalik, \& Torna, 2017).

\section{METODOLOGIA}

Ao longo deste texto, serão comparados o PSI 20 TR (Total Return) e cinco fundos de investimento mobiliário em ações portuguesas harmonizados ${ }^{3}$ : BPI PORTUGAL (PTYPIGLM0000), CAIXAGEST AÇÕES PORTUGAL (PTYCXNLP0004), SANTANDER AÇÕES PORTUGAL (PTYSAFLM0006), IMGA AÇÕES PORTUGAL (PTAFIALM0006) e NB PORTUGAL (PTYESYLM0009).

3 Com base na APFIPP (http://www.apfipp.pt/index2.aspx?MenuCode=FIM), um F.I.M. em ações portuguesas harmonizado é "um património autónomo que resulta da agregação e aplicação de poupanças de entidades individuais e coletivas em mercados primários e/ou secundários de valores. Dentro destes, os Fundos de Investimento Mobiliário (...) efetuam as suas aplicações fundamentalmente em valores mobiliários transacionáveis, cotados ou não cotados. (...) tendo a vantagem de as suas aplicações serem acompanhadas e geridas por profissionais especializados no mercado de capitais.. Nomeadamente, "por uma Sociedade Gestora de Fundos de Investimento - S.G.F.I.M., remunerada através da comissão de gestão paga pelos Fundos sob sua gestão." Na seleção realizada, temos que os fundos são abertos ("Constituídos por unidades de participação em número variável, ou seja, o número de unidades de participação varia de acordo com a procura do mercado. Uma subscrição resulta num aumento das UP's e um resgate traduz-se numa eliminação das UP's correspondentes."), de ações nacionais ("Fundos cujo objetivo consiste na aplicação de pelo menos 2/3 da carteira em ações, (...) que investem exclusivamente em ativos emitidos por entidades residentes no espaço nacional e denominados em Euro"), cuja forma de remuneração tem como base a capitalização ("Reinvestem automaticamente os rendimentos gerados pelas respetivas carteiras, não distribuindo rendimentos.”) e harmonizados (quando cumprem com a legislação em vigor quanto a determinados parâmetros, beneficiando "de um "passaporte comunitário" para a sua comercialização."). 


\title{
Uma Análise de Performance de Cinco Fundos de Investimento Mobiliário Harmonizados de Ações Portuguesas
}

\author{
Quadro 1 - Descrição dos Fundos de Investimento Mobiliário \\ em Acções Portuguesas Harmonizados
}

\begin{tabular}{|c|c|c|c|c|}
\hline $\begin{array}{l}\text { Nome do fundo: } \\
\text { BPI PORTUGAL } \\
\text { - FUNDO } \\
\text { INVESTIMENTO } \\
\text { ABERTO DE ACÇÕES } \\
\text { Código ISIN: } \\
\text { PTYPIGLM0000 } \\
\text { Código do fundo: } 120 \\
\text { Entidade gestora: } \\
\text { BPI Gestão de Activos } \\
\text { - Sociedade Gestora de } \\
\text { Fundos de Investimento } \\
\text { Mobiliário, SA } \\
\text { Tipo de fundo: Fundos de } \\
\text { Investimento de Ações } \\
\text { Situação do fundo: Em } \\
\text { atividade } \\
\text { Data de início da } \\
\text { atividade: } 03 / 01 / 1994 \\
\text { Fundo harmonizado }\end{array}$ & $\begin{array}{l}\text { Nome do fundo: } \\
\text { CAIXAGEST ACÇÕES } \\
\text { PORTUGAL - FUNDO } \\
\text { INVESTIMENTO } \\
\text { ABERTO DE ACÇÕES } \\
\text { Código ISIN: } \\
\text { PTYCXNLPO004 } \\
\text { Código do fundo: } 319 \\
\text { Entidade gestora: } \\
\text { Caixagest - Técnicas de } \\
\text { Gestão de Fundos, SA } \\
\text { Tipo de fundo: Fundos de } \\
\text { Investimento de Ações } \\
\text { Situação do fundo: Em } \\
\text { atividade } \\
\text { Data de início da } \\
\text { atividade: } 20 / 06 / 1996 \\
\text { Fundo harmonizado }\end{array}$ & $\begin{array}{l}\text { Nome do fundo: } \\
\text { FUNDO DE } \\
\text { INVESTIMENTO } \\
\text { MOBILIÁRIO ABERTO } \\
\text { SANTANDER ACÇÕES } \\
\text { PORTUGAL } \\
\text { Código ISIN: } \\
\text { PTYSAFLM0006 } \\
\text { Código do fundo: } 143 \\
\text { Entidade gestora: } \\
\text { Santander Asset } \\
\text { Management - Sociedade } \\
\text { Gestora Fundos } \\
\text { Investimento Mobiliário, } \\
\text { SA } \\
\text { Tipo de fundo: Fundos de } \\
\text { Investimento de Ações } \\
\text { Situação do fundo: Em } \\
\text { atividade } \\
\text { Data de início da } \\
\text { atividade: } 12 / 07 / 1993 \\
\text { Fundo harmonizado }\end{array}$ & $\begin{array}{l}\text { Nome do fundo: } \\
\text { IMGA ACÇÕES } \\
\text { PORTUGAL - FUNDO } \\
\text { DE INVESTIMENTO } \\
\text { ABERTO DE ACÇÕES } \\
\text { Código ISIN: } \\
\text { PTYFIALM0006 } \\
\text { Código do fundo: } 109 \\
\text { Entidade gestora: } \\
\text { IM Gestão de Activos } \\
\text { - Sociedade Gestora de } \\
\text { Fundos de Investimento, } \\
\text { SA } \\
\text { Tipo de fundo: Fundos de } \\
\text { Investimento de Ações } \\
\text { Situação do fundo: Em } \\
\text { atividade } \\
\text { Data de início da } \\
\text { atividade: } 20 / 07 / 1995 \\
\text { Fundo harmonizado }\end{array}$ & $\begin{array}{l}\text { Nome do fundo: } \\
\text { NB PORTUGAL } \\
\text { ACÇÕES - FUNDO } \\
\text { INVESTIMENTO } \\
\text { ABERTO DE ACÇÕES } \\
\text { NACIONAIS } \\
\text { Código ISIN: } \\
\text { PTYESYLM0009 } \\
\text { Código do fundo: } 414 \\
\text { Entidade gestora: } \\
\text { GNB - Sociedade } \\
\text { Gestora de Fundos de } \\
\text { Investimento Mobiliário, } \\
\text { SA } \\
\text { Tipo de fundo: Fundos de } \\
\text { Investimento de Ações } \\
\text { Situação do fundo: Em } \\
\text { atividade } \\
\text { Data de início da } \\
\text { atividade: } 15 / 09 / 1997 \\
\text { Fundo harmonizado }\end{array}$ \\
\hline
\end{tabular}

Fonte: CMVM

Primeiro ao nível do VaR e, subsequentemente, de acordo com o CAPM (Capital Asset Pricing Model), na versão Zero Beta Model, (Sharpe, 1973), em que substituímos a taxa de juro isenta de risco (ou de muito reduzido risco) pela taxa EURIBOR a 3 meses, dado que se pretende relaxar a hipótese de que os investidores podem pedir emprestado e emprestar à taxa isenta de risco ${ }^{4}$, e de algumas medidas de performance

4 Este modelo baseia-se no conceito de beta nulo (onde um gestor pode ter 100\% de posições longas e 100\% de posições curtas, ganhando apenas a taxa de juro sem risco), sem correlação com o mercado. Devido a essa liberdade de posições curtas e longas, a utilização da Euribor é justificável, pois serve de base a essas operações. Por outro lado, a tradicional taxa de juro sem risco está em "crise", fruto das crises das dívidas soberanas e trata-se de um conceito com pouca aderência à realidade. A utilização de uma taxa de juro curto prazo acaba por ser um sinal da insegurança, pois curto prazo é normalmente sinónimo de menor risco. Ao utilizar a Euribor (e por conseguinte o zero beta model) assume-se que não há constrangimentos de posições curtas e de utilização de alavancagem nos fundos analisados, o que não é exato. De qualquer forma, como a base de comparação é a mesma, e pressupondo que os fundos têm uma política de investimento similar, tal não deverá prejudicar a análise. O horizonte temporal escolhido para a Euribor (3 meses) teve como pressuposto que, no mínimo, os gestores do fundo efetuarão uma recomposição da sua carteira trimestralmente. 
extraíveis do respetivo modelo ${ }^{5}$. As fontes utilizadas foram:

European Money Markets Institute - Taxa Euribor a 3 meses $^{6}$;

Banco de Portugal - Índice de Cotações de Ações PSI Geral';

Reuters - Índice de Cotações de Ações PSI 20 TR (Total Return);

CMVM - F.I.M. em ações portuguesas harmonizados ${ }^{8}$.

O período utilizado para o VAR foram as 1.001 últimas observações com a data término a ser fixada em 28/04/17. Relativamente ao período selecionado para o CAPM, considerou-se cinco anos, designadamente entre 02/05/12e 28/04/17 $(\mathrm{n}=1.240)$. Como alguns fundos não tinham cotações divulgadas em determinadas datas, optou-se por eliminar os dados para todas as variáveis relativamente a essas, o que se traduziu numa perda residual de informação.

O benchmark adotado foi o PSI TR, uma vez que os coeficientes de correlação entre os F.I.M. e o primeiro apresentaram valores superiores face aos do PSI Geral, com exceção do F.I.M. da IMGA. Esta evidência empírica deve-se ao facto da dimensão das sociedades que compõem o índice PSI-20 ser superior à das que dele estão excluídas, levando a que os gestores dos fundos deem primazia às primeiras, por motivos defensivos, entre outros.

Para recolher as cotações dos F.I.M. selecionados, não foi possível efetuar o descarregamento direto dos dados, uma vez que à data a CMVM não tinha essa informação para tal disponível. Deste modo, fomos forçados a copiar e a colar todos os dados manualmente do website da CMVM, processo que, além de moroso, está sujeito a enfermar de erros com maior probabilidade.

Em síntese, recolhemos uma quantidade muito significativa de dados para os objetivos delineados e tratámo-los através do Microsoft Excel ${ }^{9}$, com vista a comparar as performances dos fundos, tendo tentado igualmente responder a questões relevantes que normalmente não são ponderadas por pequenos investidores quando escolhem a alocação dos seus recursos financeiros.

5 O fundo IMGA AÇÕES PORTUGAL (PTAFIALM0006) apresenta valores muito discrepantes relativamente ao Alfa de Jensen e Rácio de Treynor.

6 https://www.emmi-benchmarks.eu/euribor-org/euribor-rates.html

7 https://www.bportugal.pt/Mobile/BPStat/Serie.aspx?IndID=122814\&SerID=1151429\&Show=1\&

$\underline{\mathrm{SW}=1920}$

8 http://web3.cmvm.pt/sdi/fundos/app/pesquisa nome fundos.cfm

9 Os dados podem ser descarregados em http://henriqueamaraldias.wixsite.com/economist/publicacoes, pressionando "Dados F.I.M". 
Uma Análise de Performance de Cinco Fundos de Investimento Mobiliário Harmonizados de Ações Portuguesas

\section{VaR}

Não obstante as críticas relativas a este conceito, este é ainda hoje amplamente utilizado. A sintética explanação teórica que se segue baseia-se na obra Options, Futures, and Other Derivatives de John C. Hull (2012). O VaR tem como objetivo produzir a seguinte afirmação: “Tem-se $X$ por cento de certeza que não ocorrerá uma perda superior a $V$ unidades monetárias nos próximos $N$ dias." A variável $V$ é o VaR da carteira. É uma função de dois parâmetros: o horizonte temporal ( $N$ dias) e o nível de confiança $(X \%)$. É, portanto, o nível de perda durante o período de $N$ dias cuja probabilidade de ser excedida é de apenas (100- X\%). O VaR assume que a variação do valor da carteira (portfólio) segue uma função de distribuição de probabilidade normal ${ }^{10}$. Na sua essência, responde à questão: "How bad can things get?". Contudo, e apesar dos gestores de uma carteira tentarem encontrar uma composição para a mesma que siga essa função de distribuição de probabilidade, pode acontecer que a "cauda" da distribuição tenha um potencial de perda superior ao esperado, evidenciando um comportamento anómalo. Consequentemente, a questão passaria a ser "If things do get bad, how much can the company expect to lose?", i.e. a perda esperada durante um período de $N$ dias condicionada à ocorrência de um evento na "cauda" da distribuição correspondente a (100- X\%). Habitualmente, os analistas começam por fixar $\mathrm{N}=1$. No entanto, a fórmula geral é

$$
\text { VaR de } N-\text { dias }=\text { VaR de } 1-\text { dia } \times \sqrt{N} \text {. }
$$

Ela assume que as variações do valor de uma carteira em dias sucessivos têm distribuições normais idênticas e independentes com média zero. Com vista a calcular o VaR, vamos seguir a via da Simulação Histórica [Distribuição Empírica] que consiste em: i) criar uma base de dados dos movimentos diários das variáveis de mercado; ii) pressupor que a primeira simulação assume que a alteração percentual nas variáveis de mercado é igual à variação do primeiro dia da base de dados, que a segunda simulação assume que a alteração percentual nas variáveis de mercado é igual à variação do segundo dia da base de dados e assim sucessivamente.

Utilizou-se uma base de dados real com 1.001 dias. Se $v i$ for valor da variável

10 A distribuição Normal ou Gaussiana é muito utilizada em análises estatísticas. É uma distribuição simétrica em torno da sua média e em forma de sino. Depende de dois parâmetros que são a média e a variância da distribuição. $\mathrm{X} \sim \mathrm{N}(\mu, \sigma)$ significa que $\mathrm{X}$ tem distribuição Normal com média $\mu$ e desvio padrão $\sigma$. 
de mercado no dia $i$, podem calcular-se 1.000 simulações para o valor da variável selecionada. $\mathrm{O}$ valor do $i^{i e s i m o}$ cenário é igual a $v_{n} \times \frac{v_{i}}{v_{i-1}}$. Para tornar a interpretação dos dados mais imediata, partiu-se do valor base 100 para cada ativo, sendo também assim as perdas/ganhos percentagens. Após os cálculos elaborados, as principais conclusões são:

$1^{\text {a }}$ - Os histogramas das perdas associadas aos cenários para o PSI 20 TR e os F.I.M. assemelham-se, ainda que enviesadamente, a uma distribuição normal, pelo que a fórmula geral será uma apenas razoável aproximação.

Figura 1 - Histogramas do PSI 20 TR e dos Fundos de Investimento Mobiliário em Acções Portuguesas Harmonizados

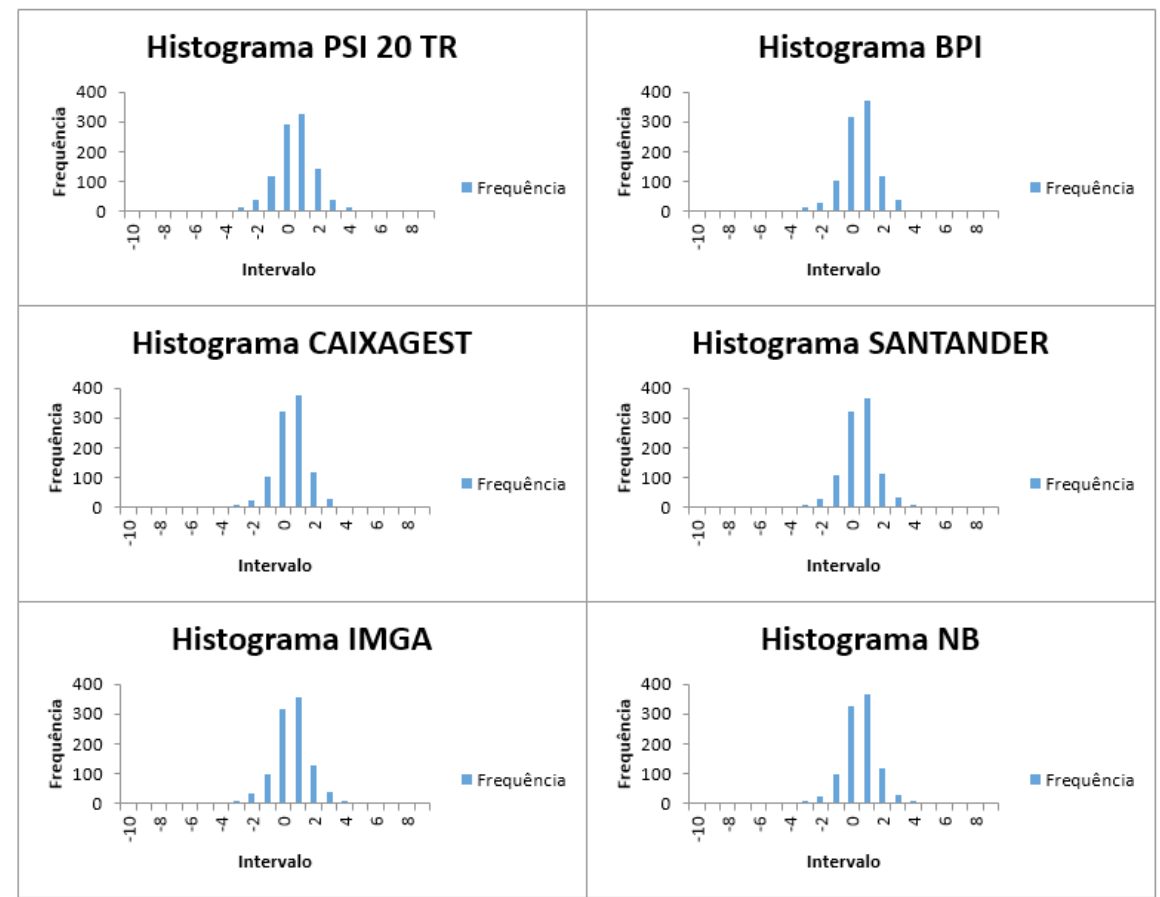

Fonte: Elaboração própria a partir dos dados da CMVM e da Reuters 
Uma Análise de Performance de Cinco Fundos de Investimento Mobiliário Harmonizados de Ações Portuguesas

$2^{\mathrm{a}}$ - Os VaR obtidos para o índice e os F.I.M. são os seguintes:

Quadro 2 - VaR dos Fundos de Investimento Mobiliário em Acções Portuguesas Harmonizados e do PSI 20 TR

\begin{tabular}{|c|c|c|c|c|c|}
\hline \multicolumn{2}{|c|}{ Var } & $\begin{array}{c}\text { 1-day 99\% } \\
\text { VaR }\end{array}$ & $\begin{array}{c}\text { 10-day 99\% } \\
\text { VaR }\end{array}$ & $\begin{array}{c}\text { 20-day 99\% } \\
\text { VaR }\end{array}$ & $\begin{array}{c}\text { 30-day 99\% } \\
\text { VaR }\end{array}$ \\
\hline 1 & NB PORTUGAL & $-3,615009$ & $-11,4317$ & $-16,1668$ & $-19,8002$ \\
\hline 2 & IMGA & $-3,747152$ & $-11,8495$ & $-16,7578$ & $-20,5240$ \\
\hline 3 & BPI & $-3,769323$ & $-11,9196$ & $-16,8569$ & $-20,6454$ \\
\hline 4 & PSI TR & $-3,809522$ & $-12,0468$ & $-17,0367$ & $-20,8656$ \\
\hline 5 & SANTANDER & $-3,818151$ & $-12,0741$ & $-17,0753$ & $-20,9129$ \\
\hline 6 & CAIXAGEST & $-3,927393$ & $-12,4195$ & $-17,5638$ & $-21,5112$ \\
\hline
\end{tabular}

Fonte: Elaboração própria a partir dos dados da CMVM e da Reuters

Aquele que apresenta um menor VaR para 1, 10, 20 e 30 dias é o F.I.M. do NB. A interpretação, por exemplo, do VaR a 1-dia do NB é: no próximo dia, a carteira deste F.I.M. terá uma probabilidade de $1 \%$ de ter uma perda superior a 3,615009\%.

$3^{a}$ - O cenário em que ocorreu a pior perda foi o 790, que corresponde ao dia 24 de junho de 2016, que foi precisamente o dia seguinte ao do referendo realizado no Reino Unido e que teve como resultado a decisão de saída deste da União Europeia, o que gerou algum pânico, nos primeiros momentos, nos mercados de capitais. Pergunta-se por que razão os gestores, sabendo da incerteza do outcome do referendo, não assumiram posições curtas. Julgamos que é pelo facto do mercado português estar longe de ser eficiente, não permitindo aos gestores assumirem posições táticas muito diferentes da estratégia global. $\mathrm{O}$ mercado português não permite aproveitar da mesma forma as ineficiências detetadas na posição longa e na posição curta (onde tradicionalmente as ineficiências são maiores).

\section{Capital Asset Pricing Model (CAPM)}

A seguinte abordagem teórica apoia-se na obra O Governo das Sociedades e os Investidores Institucionais: Disponibilidade, Condicionantes e Implicações de Carlos Alves (2005). O objetivo deste estudo empírico é poder aferir se o desempenho dos gestores dos F.I.M. pode ser considerado como neutral, supranormal ou infranormal 
relativamente ao benchmark escolhido (PSI 20 TR), mas também comparar a performance interpares. Segundo o autor:

"O CAPM descreve o retorno de equilíbrio de cada ativo em função do seu comportamento perante variações do retorno do mercado (...)", sendo "visto como um modelo para avaliação de ativos financeiros no quadro de um ambiente competitivo, onde o retorno de cada título é determinado em função do seu contributo marginal (...) para uma carteira eficiente de ativos. Combinando numa tal carteira de ativos com risco e um ativo isento de risco, o CAPM reconduz-se a uma forma linear e permite avaliar a rendibilidade esperada para cada ativo. Essa linha, a que se dá a designação de Security Market Line (SML), é a expressão de síntese do CAPM, e é descrita por uma equação, como a que se segue:

$$
E\left(R_{p, t}\right)=R_{f}+\beta_{p}\left[E\left(R_{m, t}\right)-R_{f}\right]
$$

com

$$
\beta_{p}=\frac{\operatorname{Cov}_{p, m}}{\sigma_{m}^{2}} \quad \text { [3.2] }
$$

onde: $E\left(R_{p, t}\right)$ é o retorno esperado do ativo ou da carteira p para o período t; $R_{f}$ é a taxa de retorno para ativos isentos de risco; $\beta_{p}$ mede a resposta do ativo a movimentos no retorno do portfólio do mercado, ou seja, mede o risco sistemático do ativo ou da carteira; $E\left(R_{m, t}\right)$ é o retorno esperado para a carteira do mercado para o período t; $\operatorname{Cov}_{p, m}$ simboliza a covariância entre a taxa de retorno da carteira do mercado e a taxa de retorno de p; e $\sigma_{m}^{2}$ é a variância da taxa de retorno da carteira do mercado." (Alves, 2005)

Os principais pressupostos do CAPM são: (1) Os investidores necessitam apenas de conhecer os retornos esperados, as variâncias, e as covariâncias dos retornos para determinar que carteiras são ótimas para eles; (2) Os investidores têm expectativas homogéneas quanto aos retornos esperados, as variâncias e correlações de todos os ativos; (3) Os investidores podem comprar e vender os ativos em qualquer quantidade (divisibilidade perfeita) sem afetar o preço, e todos os ativos são transacionáveis; (4) Toda a informação está disponível para qualquer investidor, de forma instantânea e gratuita; 
(5) Os investidores podem pedir emprestado e emprestar à taxa de juro sem risco sem limite, e podem vender a descoberto (short selling) qualquer ativo em qualquer atividade; (6) Os investidores não pagam impostos, nem existem custos de transação.

Deste modelo conclui-se que em equilíbrio todos os ativos se posicionam sobre a SML (Security Market Line - SML). Como já referido, optámos por utilizar o Zero Beta Model, pelas razões já apontadas na nota de rodapé 4, (Sun \& Yang, 2003; Sharpe, 1973). Assim, $E\left(R_{p, t}\right)=E\left(R_{Z}\right)+\beta_{p}\left[E\left(R_{m, t}\right)-E\left(R_{Z}\right)\right]$.

As medidas de performance utlizadas, para além do retorno efetivo (em média aritmética e geométrica) e do retorno esperado por aplicação do CAPM na sua forma não standard, foram o Alfa de Jensen ${ }^{11}$, Rácio de Treynor ${ }^{12}$ e Rácio de Sharpe ${ }^{13}$.

\section{I - Análise dos retornos}

Quadro 3 a - Retornos em média aritmética dos Fundos de Investimento Mobiliário em Acções Portuguesas Harmonizados e do PSI 20 TR

\begin{tabular}{|c|c|c|c|c|c|}
\hline \multirow{2}{*}{$\begin{array}{c}\text { Retorno Efetivo } \\
\text { (média aritmética) }\end{array}$} & \multicolumn{2}{c|}{ Retorno } & \multicolumn{2}{c|}{ Face PSI TR } \\
\cline { 3 - 6 } & Diário & Anualizado & Diário & Anualizado \\
\hline 1 & SANTANDER & $0,03841 \%$ & $9,99275 \%$ & $0,01804 \%$ & $4,81116 \%$ \\
\hline 2 & BPI & $0,03703 \%$ & $9,61770 \%$ & $0,01666 \%$ & $4,43610 \%$ \\
\hline 3 & IMGA & $0,03577 \%$ & $9,27540 \%$ & $0,01540 \%$ & $4,09380 \%$ \\
\hline 4 & CAIXAGEST & $0,03319 \%$ & $8,57782 \%$ & $0,01282 \%$ & $3,39622 \%$ \\
\hline 5 & NB PORTUGAL & $0,02967 \%$ & $7,63340 \%$ & $0,01929 \%$ & $2,45180 \%$ \\
\hline
\end{tabular}

Fonte: Elaboração própria a partir dos dados da CMVM e da Reuters

11 Jensen (1968), propôs a medida conhecida por “alfa de Jensen", que corresponde à diferença entre a rendibilidade obtida pela carteira avaliada e a rendibilidade que, nos termos do equilíbrio definido pelo CAPM, essa carteira deveria ter obtido. É importante salientar que a avaliação da performance é realizada com base em informação ex-post, e não com base no retorno esperado ex-ante subjacente à formulação, uma vez que o objetivo é avaliar o comportamento efetivo do gestor: .

12 Treynor (1966) também considera o risco sistemático como indicador relevante do risco assumido, pelo que se reconduz de novo ao plano formado pelo eixo representativo da rendibilidade média e pelo eixo representativo do nível de risco sistemático: . Em que no denominador tem-se a quantidade de risco sistemático da carteira e no numerador o excesso de retorno da mesma, podendo interpretar-se como o excesso de retorno por unidade de risco sistemático. Quanto maior este rácio for, tal indica que se criou mais riqueza por unidade de risco sistemático.

13 A medida de Sharpe (1994) é em tudo idêntica à medida de Treynor, com a única diferença de que o conceito de normalidade é aqui representado pela reta Capital Market Line (CML), que passa pelo ponto representativo da carteira de mercado e pelo ponto representativo do ativo isento de risco no plano delimitado pela rendibilidade média e pelo desvio padrão do retorno. Trata-se, portanto, do excesso de retorno por unidade de risco total. 
Quadro 3 b - Retornos em média geométrica dos Fundos de Investimento Mobiliário em Acções Portuguesas Harmonizados e do PSI TR

\begin{tabular}{|c|c|c|c|c|c|}
\hline \multirow{2}{*}{$\begin{array}{c}\text { Retorno Efetivo } \\
\text { (média aritmética) }\end{array}$} & \multicolumn{2}{c|}{ Retorno } & \multicolumn{2}{c|}{ Face PSI TR } \\
\cline { 3 - 6 } & Diário & Anualizado & Diário & Anualizado \\
\hline 1 & SANTANDER & $0,03062 \%$ & $7,88822 \%$ & $0,01900 \%$ & $4,96378 \%$ \\
\hline 2 & BPI & $0,02988 \%$ & $7,69120 \%$ & $0,01826 \%$ & $4,76676 \%$ \\
\hline 3 & IMGA & $0,02817 \%$ & $7,23611 \%$ & $0,01655 \%$ & $4,31167 \%$ \\
\hline 4 & CAIXAGEST & $0,02665 \%$ & $6,83074 \%$ & $0,01502 \%$ & $3,90631 \%$ \\
\hline 5 & NB PORTUGAL & $0,02270 \%$ & $5,79121 \%$ & $0,01108 \%$ & $2,86678 \%$ \\
\hline
\end{tabular}

Fonte: Elaboração própria a partir dos dados da CMVM e da Reuters

\section{II - Aplicação do CAPM}

Quadro 4 - Dados do CAPM para os Fundos de Investimento Mobiliário em Acções Portuguesas Harmonizados

\begin{tabular}{|c|c|c|c|c|c|c|c|}
\hline \multicolumn{2}{|c|}{ CAPM } & \multirow{2}{*}{ Covariância } & \multirow{2}{*}{ Beta } & \multicolumn{2}{c|}{ E(R) } & \multicolumn{2}{c|}{ Face PSI TR } \\
\cline { 5 - 8 } & & & Diário & Anualizado & Diário & Anualizado \\
\hline 1 & SANTANDER & 0,00015 & 0,88061747 & $0,01494 \%$ & $3,77460 \%$ & $-0,00543 \%$ & $-1,40700 \%$ \\
\hline 2 & BPI & 0,00015 & 0,86314565 & $0,01415 \%$ & $3,57026 \%$ & $-0,00623 \%$ & $-1,61134 \%$ \\
\hline 3 & NB PORTUGAL & 0,00015 & 0,84573023 & $0,01335 \%$ & $3,36698 \%$ & $-0,00702 \%$ & $-1,81462 \%$ \\
\hline 4 & CAIXAGEST & 0,00014 & 0,82313775 & $0,01233 \%$ & $3,10387 \%$ & $-0,00805 \%$ & $-2,07773 \%$ \\
\hline 5 & IMGA & 0,00002 & 0,11801699 & $-0,01975 \%$ & $-4,78118 \%$ & $-0,04013 \%$ & $-9,96278 \%$ \\
\hline
\end{tabular}

Fonte: Elaboração própria a partir dos dados da CMVM e da Reuters

\section{III - Alfa de Jensen}

Quadro 5 a - Alfa de Jensen dos Fundos de Investimento Mobiliário em Acções Portuguesas Harmonizados

\begin{tabular}{|c|c|c|c|}
\hline \multirow{2}{*}{ Alfa de Jensen } & \multicolumn{2}{c|}{ Alfa de Jensen } \\
\cline { 3 - 4 } & Diário & Anualizado \\
\hline 1 & IMGA & 0,00056 & $14,75928 \%$ \\
\hline 2 & SANTANDER & 0,00023 & $5,99290 \%$ \\
\hline 3 & BPI & 0,00023 & $5,83982 \%$ \\
\hline 4 & CAIXAGEST & 0,00021 & $5,30984 \%$ \\
\hline 5 & NB PORTUGAL & 0,00016 & $4,12801 \%$ \\
\hline
\end{tabular}

Fonte: Elaboração própria a partir dos dados da CMVM e da Reuters 
Uma Análise de Performance de Cinco Fundos de Investimento Mobiliário Harmonizados de Ações Portuguesas

\section{IV - Rácio de Treynor e Rácio de Sharpe}

Quadro 5 b - Rácio de Treynor e Rácio de Sharpe dos Fundos de Investimento Mobiliário e m Acções Portuguesas Harmonizados

\begin{tabular}{|c|c|c|}
\hline \multicolumn{3}{|c|}{ Rácio de Treynor } \\
\hline 1 & IMGA & 0,005160 \\
\hline 2 & SANTANDER & 0,000721 \\
\hline 3 & BPI & 0,000720 \\
\hline 4 & CAIXAGEST & 0,000708 \\
\hline 5 & NB PORTUGAL & 0,000648 \\
\hline
\end{tabular}

\begin{tabular}{|c|c|c|}
\hline \multicolumn{3}{|c|}{ Rácio de Sharpe } \\
\hline 1 & BPI & 0,052043 \\
\hline 2 & CAIXAGEST & 0,051049 \\
\hline 3 & SANTANDER & 0,051016 \\
\hline 4 & IMGA & 0,049465 \\
\hline 5 & NB PORTUGAL & 0,046491 \\
\hline
\end{tabular}

Fonte: Elaboração própria a partir dos dados da CMVM e da Reuters

\section{CONCLUSÕES}

Os métodos utilizados, VaR e CAPM, exibem as limitações já apontadas (entre outras). Todavia, os investigadores reconhecem a sua utilidade dentro de determinadas condições e para determinados fins, continuando a aperfeiçoá-los tecnicamente.

Os coeficientes de determinação entre os F.I.M. e o benchmark considerado (PSI TR) confirmaram as previsões do CAPM, com exceção do IGMA. Não havendo meios de saber quais são os ativos específicos que compõem a carteira deste último, podemos supor que nesta estão contidas ações de outras empresas que não fazem parte do benchmark.

Como referido e tal como a literatura recomenda, o Rácio de Sharpe é dominante face aos outros que considerámos. Assim, o F.I.M. do BPI é o que exibe uma maior rendibilidade por unidade de risco total.

O Rácio de Sharpe pode servir para confirmar o Alfa de Jensen. Mas neste caso, o peso do risco total influenciou decisivamente os resultados.

Uma análise que se focasse apenas na rendibilidade supranormal, ignorando o risco, optaria pelo F.I.M. do Santander (pelas razões já apontadas desconsiderámos o F.I.M. do IGMA), cometendo-se o erro, a que os investidores continuam a ser propensos, de menosprezar a dimensão risco. Tendência que se agrava com yields tão historicamente reduzidas como as verificadas no mercado de dívida. 
$\mathrm{O}$ VaR revela-nos que, para o período fixado, as perdas seriam relativamente similares para todos os F.I.M., existindo, por exemplo, uma diferença de $-4,094 \%$ entre o do NB PORTUGAL e o do BPI para o critério VaR a 1-dia.

Futuramente, será interessante comparar os resultados ora obtidos com os de outras variantes do VaR e do CAPM.

Este trabalho pretendeu evidenciar a performance de F.I.M. cujos ativos são ações portuguesas sob diversas perspetivas.

Dada alguma falta de informação e de transparência com a que a maioria dos investidores se deparam, é urgente que as entidades reguladoras e supervisoras dos mercados e do sistema financeiro invistam recursos mais significativos na literacia financeira dos cidadãos, permitindo uma maior racionalidade na tomada de decisão. Esperamos, por isso, ter contribuído para uma melhor perceção do mercado.

\section{REFERÊNCIAS}

Alves, C. (2005). O Governo das Sociedades e os Investidores Institucionais: Disponibilidade, Condicionantes e Implicações. Coimbra: Almedina.

Blyth, M. (2013). Austeridade - A História de Uma Ideia Perigosa. Lisboa: Quetzal Editores.

Cícero, M. T. (45 a.C. /1923). De Divinatione. Harvard: Loeb Classical Library.

DeYoung, R., Kowalik, M., \& Torna, G. (2017). Private Equity Investment in U.S. Banks. Recuperado em 17 de novembro, 2017 de https://editorialexpress.com/cgi-bin/ conference/download.cgi? db name=XXVIfinanceforum\&paper $\_$id $=104$

Dowd, K., Cotter, J., Humphrey, C., \& Woods, M. (2008). How Unlucky is 25-Sigma? Journal of Portfolio Management 34 (4), 76-80. doi: https://doi.org/10.3905/ jpm.2008.709984

Fama, E. F., \& French, K. R. (2004). The Capital Asset Pricing Model: Theory and Evidence. Journal of Economic Perspetives 18(3), 25-46.

Hull, J. C. (2012). Options, Futures, and Other Derivatives. Eighth Edition. Boston: 
Uma Análise de Performance de Cinco Fundos de Investimento Mobiliário Harmonizados de Ações Portuguesas

Prentice Hall.

Hurlin, C., \& Tokpavi, S. (2007). Backtesting Value-at-Risk Accuracy: A New Simple Test. Journal of Risk 9(2), 19-37. doi: https://doi.org/10.21314/JOR.2007.148

Jensen, M. C. (1968). The Performance of Mutual Funds in the Period 1945-1964. Journal of Finance 23, 389-416. doi: https://doi.org/10.1111/j.1540-6261.1968. $\underline{\text { tb00815.x }}$

Lintner, J. (1965). The valuation of risk Assets and the Selection of Risky Investments in Stock Portfolios and Capital Budgets. Review of Economics and Statistics, 47 (1), 13-37. doi: https://doi.org/10.2307/1924119

Markowitz, H. M. (1952). Portfolio Selection. The Journal of Finance 7(1), 77-91. doi: https://doi.org/10.1111/j.1540-6261.1952.tb01525.x

Merton, R. C. (1973). An Intertemporal Capital Asset Pricing Model. Econometrica 41(5), 867-887. doi: https://doi.org/10.2307/1913811

Mitrodima, G., \& Oberoi, J. (2017). Value at Risk models of autoregressive quantiles of improved performance on financial criteria. Recuperado em 17 de novembro de $2017 \mathrm{de}$ https://papers.ssrn.com/sol3/papers.cfm?abstract id=2649348

Sharpe, W. F. (1964). Capital Asset Prices: A Theory of Market Equilibrium under Conditions of Risk. Journal of Finance 19(3), 425-42. doi: https://doi. org/10.1111/j.1540-6261.1964.tb02865.x

Sharpe, W. (1973). The Capital Asset Pricing Model: Traditional and 'Zero-Beta' Versions. Journal of the Midwest Finance Association, 1-12.

Sharpe, W. F. (1994). The Sharpe Ratio, The Journal of Portfolio Management 21 (1), 49-58. doi: https://doi.org/10.3905/jpm.1994.409501

Sun, N., \& Yang, Z. (2003). Existence of Equilibrium and Zero-Beta Pricing Formula in the Capital Asset Pricing Model with Heterogeneous Beliefs. Annals of Economics and Finance 4, 51-71.

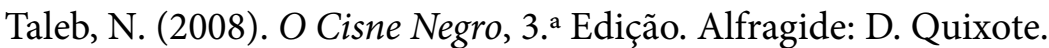


Treynor, J. L. (1966). How to rate management investment funds, Harvard Business Review 43(1) (January-February), 63-75.

Youngman, P. (2009). Procyclicality and Value at Risk. Reports Bank of Canada, junho 2009, 51-54. 